\title{
CURATIVOS PARA TRATAMENTO DE FERIDAS OPERATÓRIAS ABDOMINAIS: UMA REVISÃO SISTEMÁTICA
}

\author{
Carolina Giordani SILVA ${ }^{a}$, Maria da Graça Oliveira CROSSETTI ${ }^{b}$
}

\section{RESUMO}

O objetivo deste estudo foi identificar o curativo prevalente para o tratamento das feridas operatórias abdominais com complicações, visando buscar evidências que possam subsidiar o desenvolvimento de um protocolo institucional para o tratamento das mesmas. Metodologia: desenvolveu-se uma Revisão Sistemática, que teve como pergunta norteadora "Qual é o curativo prevalente no tratamento dos pacientes com complicações de feridas operatórias (FOs) abdominais?” Foram utilizados MeSH para buscar o maior número de estudos possíveis em sete base de dados eletrônicas. Resultados: a busca nas bases de dados resultou em 6.107 artigos que, após serem submetidos aos testes de relevância, resultaram em 33 estudos que compuseram a amostra. O curativo a VAC foi o mais indicado para tratar FOs abdominais que tiveram complicações. Conclusão: sugerem-se novas pesquisas para que se possa avaliar a efetividade e viabilidade da terapia VAC na nossa realidade.

Descritores: Revisão. Curativos oclusivos. Cicatrização.

\section{RESUMEN}

El objetivo de este estudio fue identificar los curativos prevalentes en el tratamiento de heridas quirúrgicas abdominales con complicaciones, con la finalidad de obtener evidencias que puedan subsidiar el desarrollo de protocolo institucional para tratamiento de las mismas. Metodología: una Revisión Sistemática que tuvo como pregunta orientadora ¿̇Cuál es el curativo prevalente en el tratamiento de los pacientes con complicaciones de heridas operatorias abdominales? Fueron utilizados MeSH para buscar el mayor número de estudios posibles, en siete bases de datos electrónicas. Resultados: La búsqueda en las bases de datos resultó en 6107 artículos, después se realizaron los Test de Relevancia resultando la muestra final de 33 estudios. El uso del curativo VAC es curativo más indicado para tratar FOs abdominales que tuvieron complicaciones. Conclusión: Se sugieren nuevas investigaciones, para que se pueda evaluar la efectividad y viabilidad de la terapia VAC en nuestra realidad.

Descriptores: Revisión. Apósitos oclusivos. Cicatrización de heridas.

Título: Los apósitos para el tratamiento quirúrgico de las heridas abdominales: una revisión sistemática.

\section{ABSTRACT}

The aim of this study was to identify the dressings used to treat abdominal surgical wounds with complications, in order to look for evidence that supports the development of an institutional protocol for handling these wounds. Methodology: a Systematic Review was developed, which had as a guiding question: What is the prevalent dressing in the treatment of patients with complications in abdominal surgical wounds? The MeSH database was used to search for the largest possible number of studies in seven electronic databases. Results: The search in the databases resulted in 6,107 articles, after being tested for relevance, the result was 33 studies that comprised the sample. The use of the VAC dressing was the best suited to treat abdominal surgical wounds with complications. Conclusion: Further research is suggested, so that the effectiveness and feasibility of VAC therapy in our reality can be assessed.

Descriptors: Review. Occlusive dressings. Wound healing.

Title: Dressings for the treatment of abdominal surgical wounds: a systematic review.

\footnotetext{
a Enfermeira do Hospital de Clínicas de Porto Alegre, Mestre em Enfermagem pela Universidade Federal do Rio Grande do Sul, Membro do Núcleo de Estudos do Cuidado de Enfermgem - NECE, Porto Alegre, Rio Grande do Sul, Brasil.

b Professora Titular da Escola de Enfermagem da Universidade Federal do Rio Grande do Sul, Coordenadora do Núcleo de Estudos do Cuidado de Enfermagem - NECE, Porto Alegre, Rio Grande do Sul, Brasil.
} 


\section{INTRODUÇÃO}

Os prejuízos causados pelo aumento do tempo de permanência hospitalar de um paciente devido ao retardo na cicatrização de lesões de pele ou infecção de uma ferida operatória (FO) têm sido foco de preocupação em diferentes contextos das práticas de saúde ${ }^{(1)}$. Nesse sentido, em outubro de 1999, um grupo de enfermeiras do Hospital de Clínicas de Porto Alegre (HCPA), assessoradas por uma professora da Escola de Enfermagem da Universidade Federal do Rio Grande do Sul (EEUFRGS), formaram um grupo de trabalho para estudar essa problemática, do qual fiz parte de 2006 até 2009, como consultora representante do serviço de emergência.

A iniciativa do grupo foi, primeiro, estudar o perfil epidemiológico da população atendida no HCPA com lesões de pele, visando implementar protocolos de cuidados, de modo a orientar intervenções adequadas para essas lesões. Nesse contexto, o grupo dedicou-se, inicialmente, à implementação do protocolo de prevenção e tratamento da úlcera por pressão, baseado na aplicação da Escala de Braden, adaptada e validada para nossa realidade.

O protocolo não inclui o cuidado ao paciente com FO. Destaca-se que, nas prescrições médicas e de enfermagem relacionadas a realização do curativo nas FOs, há uma falta de consenso acerca desta intervenção, demonstrando que a mesma é feita a critério de cada profissional, sem considerar as condições clínicas do paciente, o contexto, a natureza e os aspectos fisiopatológicos da ferida. Além disso, não seguem uma periodicidade, nem tampouco padronização de produto e de cobertura a ser utilizado no curativo dessa lesão.

Considerando que no HCPA, em 2007, ocorreram, em média, 35.735 cirurgias $^{(2)}$, a prática profissional tem mostrado que são frequentes as complicações com FOs, justificando-se, assim, a necessidade da padronização de curativos específicos para esse tratamento, através da criação de um protocolo.

Os protocolos são padrões clínicos acordados entre membros de uma mesma organização profissional, orientando a tomada de decisão clínica, pois focam no diagnóstico e no tratamento de situações específicas $^{(3)}$. Entretanto, deve-se ressaltar que não substituem a avaliação clínica individual, pois esses exigem a integração desta com a melhor evidência clínica disponível. Portanto, o desenvolvimento de um protocolo deve estar preocupado com a coleta de informações válidas na literatura científica para auxiliar na identificação de diagnóstico, prognóstico, prevenção, terapia eficaz, intervenção ou outras questões clínicas de interesse.

Assim, considera-se que um protocolo específico para curativos de $\mathrm{FO}$ auxiliaria os profissionais no cuidado do paciente em pós-operatório, contribuindo para uma intervenção clínica mais acurada, bem como na promoção da qualidade de vida e consequente redução de custos hospitalares.

Entende-se que a pele é o maior órgão do ser humano, logo, é evidente a responsabilidade da equipe de saúde, principalmente do enfermeiro, em promover e cooperar com o organismo humano para uma perfeita reconstrução tecidual. Nesse sentido, embora a cicatrização seja um complexo processo sistêmico que exige do organismo a ativação, a produção e a inibição de grande número de componentes moleculares e celulares, responsáveis pelo processo de restauração tissular ${ }^{(1)}$, não se pode descuidar do tratamento tópico das FOs, pois este é um fator que pode colaborar ou prejudicar o organismo quanto a cicatrização.

A FO é considerada uma complicação biológica, mas que tem tendência a regressão espontânea completa, dentro de um prazo mais ou menos preestabelecido e com poucas variações individuais. Contudo, quando evidenciam-se fatores estranhos ao seu desenvolvimento normal, e que podem estar relacionados ao tipo de patologia, ao estado geral do paciente ou ao procedimento cirúrgico adotado, surgem complicações sistêmicas ou locais que merecem cuidados específicos. Dentre estas, destacam-se a infecção e a deiscência de sutura ${ }^{(4)}$.

A infecção da FO é uma séria complicação que prolonga a convalescença do paciente, aumenta os gastos e a permanência do mesmo no hospital, provocando comorbidades, problemas psicossociais e incapacitantes e que, eventualmente, evolui para uma condição fatal. A incidência de infecção depende de alguns fatores como o da natureza e da duração da cirurgia, da assepsia e da antissepsia. A cirurgia contaminada, aquela em que houve feridas traumáticas com menos de seis horas, presença de processos inflamatórios sem a presença de pus e infectada, tendem a ter um índice de infecção mais elevado. Todavia, mesmo em cirurgia limpa, em que não houve a quebra dos princípios de antissepsia e técnica cirúrgica, ela pode ocorrer por questões ligadas ao ambiente hospitalar ${ }^{(4)}$. 
Além disso, a infecção de FO constitui uma parcela significativa no total de todas as infecções hospitalares, sendo considerada a segunda causa dessas infecções ${ }^{(5)}$. Nos Estados Unidos, representam $40 \%$ de todas as infecções hospitalares entre os pacientes cirúrgicos, sendo que, aproximadamente, $3 \%$ dos pacientes em pós-operatório desenvolvem infecção de FO. Além disso, despendem $60 \%$ a mais de tempo em unidades de tratamento intensivo e, se esta for desenvolvida após alta hospitalar, esses pacientes têm cinco vezes mais probabilidades de serem readmitidos no hospital ${ }^{(5-7)}$.

Há uma associação entre a infecção da FO com a ocorrência de deiscência de sutura, a qual pode ocorrer por infecção ou pelo excesso de ressecção, com tensão no retalho, isquemia e interferência na cicatrização. A deiscência de sutura é considerada uma das complicações cirúrgicas abdominais mais graves, com índice de mortalidade de $10 \%$ e caracterizada como uma emergência cirúrgica pelo risco de evisceração, que geralmente ocorre entre o quarto e o décimo quarto dia após a cirurgia ${ }^{(8,9)}$. No entanto, é mais frequente ocorrer pelo nono dia de pós-operatório, quando a infecção da ferida cirúrgica resulta em uma variável importante para este evento, e a contaminação bacteriana da cavidade abdominal gera complicações que impedem a cicatrização por fechamento primário, aumentando as chances de deiscência de sutura ${ }^{(10)}$.

Entre as condições imediatas que mais predispõem esta deiscência de sutura estão: infecção da ferida, íleo paralítico prolongado, fechamento deficiente da parede, hematoma e acúmulo de serosidade, desnutrição hipovitaminose $\mathrm{C}$ e reoperação precoce. O episódio agudo é geralmente precipitado por esforço de tosse, vômitos, soluços prolongados ou esforço exagerado para deambulação ou evacuação( ${ }^{(6)}$.

Sendo assim, evidencia-se que o fechamento primário é a melhor cicatrização que pode ocorrer com a FO. Para que este processo transcorra sem intercorrências, o enfermeiro, a quem compete avaliar o paciente e prescrever cuidados específicos no pré e no pós-operatório, contribui, com sua competência profissional, para evitar a ocorrência de complicações.

Com esse intuito, a avaliação do sítio cirúrgico é fundamental e envolve o exame físico da ferida e da pele adjacente, relacionando os achados com o processo fisiológico da cicatrização. Este exame inclui a mensuração da incisão, observação do tecido da ferida, com atenção a reepitelização, a integridade da linha de sutura, o exsudato que por ventura possa drenar e a palpação da incisão atentando para a deposição de colágeno(11).

Nesse sentido, considera-se ser necessária a indicação de um curativo adequado para tratamento de complicações de FO abdominais. Observa-se na literatura que é incomum uma uniformidade nas condutas indicadas. Entende-se que o curativo sobre o sítio cirúrgico, dentre outras finalidades, tem a função de absorver exsudatos e deve ser usado enquanto persistir o sangramento e/ou drenagem de secreção. $\mathrm{O}$ curativo age como uma barreira contra micro-organismos exógenos, desde que sua superfície externa esteja seca. $\mathrm{O}$ tempo de permanência é questionável, mas geralmente se remove após 24 horas, deixando a ferida exposta se não há nenhuma complicação, pois a remoção precoce permite uma fácil avaliação do local e detecção de anormalidades, bem como reduz o número de horas da enfermagem para troca de curativos e os custos com materiais médico hospitalares $^{(11)}$.

Diante disso, decidiu-se, por meio deste estudo, conhecer o tipo de curativo que tem sido aplicado no tratamento de FOs complicadas, visando a busca de evidências que possam subsidiar a elaboração de um protocolo específico para o tratamento dessas lesões. Neste sentido, o objetivo deste estudo foi identificar o curativo prevalente no tratamento de FOs abdominais com complicações em pacientes com 18 anos ou mais ${ }^{(12)}$.

\section{METODOLOGIA}

O estudo consistiu em uma revisão sistemática, o qual constitui uma revisão de estudos por meio de uma abordagem sistemática, utilizando metodologia claramente definida, buscando minimizar os erros nas conclusões ${ }^{(13)}$.

A revisão sistemática inicia-se com a definição apropriada da questão terapêutica, o problema. Nessa fase, devem ser considerados os aspectos diretamente relacionados com os objetivos da revisão, tipo a definição dos participantes, intervenções a serem avaliadas e os resultados a serem mensurados, que se referem à temática em análise.

Nesse sentido, existem quatro componentes fundamentais para formulação de uma questão de pesquisa, sintetizados na sigla PICO, em que $\mathbf{P}=$ participante, $\mathbf{I}=$ intervenção, $\mathbf{C}=$ controle $\mathrm{e} \mathbf{O}=$ desfecho $^{(14)}$. 
No mínimo dois desses componentes, a saber, o P e I são obrigatórios para a sistematização da pergunta de pesquisa.

A pergunta que norteou a revisão sistemática da literatura neste estudo foi: Qual é o curativo prevalente no tratamento dos pacientes com complicações de feridas operatórias abdominais?

Dessa forma temos: $\mathbf{P}=$ paciente cirúrgico com idade igual ou maior que 18 anos, $\mathbf{I}=$ tipo de curativo utilizado para o tratamento de FOs abdominais e $\mathbf{O}=$ número de dias para a cicatrização.

Foram consultadas sete bases eletrônicas, a saber: National Library of Medicine (Medline), Literatura Latino- Americana e do Caribe em Ciências da Saúde (Lilacs), Cochrane Collaboration, Web of Science, Cumulative Index to Nursing and Allied Health Literature (CINAHL), Scientific Electronic Library Online (Scielo) e SciVerse Scopus. A escolha por estas bases de dados deveu-se ao fato de que as mesmas englobam publicações nacionais e internacionais, em um amplo espectro, e o maior número de periódicos indexados.

Definimos os MeSH para buscar o maior número de artigos possíveis, sendo utilizados os controlados, quais sejam: surgical wound dehiscence, surgical wound infection, abdomen. Para ampliar a seleção das produções, também foram utilizados MeSH não controlados: surgical wound care, surgical wound dressing, surgical wound management, surgical wound healing, wound dressing, surgical wound; dressing, abdominal surgical wound infection. Além disso, foi utilizado a conexão entre os $\mathrm{MeSH}$ através do operador booleano AND.

Os critérios de inclusão desta revisão sistemática foram: artigos primários do tipo: ensaios clínicos randomizados, estudo de coorte, caso controle; publicados no período de 1998 a 2010, em idiomas inglês, português e espanhol; acesso on line livres; que envolveram cirurgias abdominais eletivas e foram realizados com população com idade igual ou maior que 18 anos.

É importante ressaltar que, mesmo utilizando os descritores preconizados pelo $\mathrm{MeSH}$, é comum obter referências de estudos irrelevantes para a pesquisa. Neste sentido, os artigos selecionados foram submetidos a testes de relevância para refinar a seleção e definir a amostra, conforme figura 1 .

A busca dos artigos nas bases de dados, segundo os MeSHs controlados e não controlados, resultou em 6.107 produções. Este processo de seleção foi bastante elaborado, pois foi realizada estratégia de busca ampla, a fim de adquirir um número expressivo de estudos.

Nesta etapa, ao fazer a busca dos dados nas diferentes bases eletrônicas, a pesquisadora realizou o Teste de Relevância Preliminar, o qual consistiu na exclusão dos artigos que, por meio do título e do resumo breve apresentado nos resultados das buscas em cada base, já demonstravam-se irrelevantes para atender a questão norteadora desta revisão, sendo excluídos 5.578 artigos. Foram identificados 103 repetidos, sendo que alguns constaram mais de duas vezes na coleta de dados. Após serem submetidos ao Teste Preliminar, foram selecionados 426 artigos para passarem pelo Teste de Relevância I.

O Teste de Relevância I consistiu na avaliação dos resumos dos estudos que foram selecionados no teste preliminar. Nesta etapa, foram excluídos 372 artigos, pois não estavam em consonância com a questão norteadora. Ao final do teste de relevância I, 54 produções foram considerados adequados para prosseguir no estudo.

Seguiu-se, então, para a quarta etapa, a qual consistiu na avaliação dos estudos na íntegra através da submissão ao Teste de Relevância II, em que se avaliou a qualidade metodológica, a relevância e a adequação dos resultados em atenção à questão norteadora desta revisão. Nesta etapa, foram excluídos 19 artigos que possuíam nível de evidência e qualidade metodológica ruim por se tratarem de estudos de casos. Ao final do teste de relevância II, 35 artigos foram considerados adequados para prosseguir no estudo.

Seguiu-se, então, para submissão dos artigos ao Teste de Relevância III ou Teste de Relevância Final, em que se avaliou a qualidade metodológica, minuciosamente, em relação ao objetivo do estudo, analisando se este tinha relação com a questão norteadora da revisão sistemática; se a metodologia empregada estava suficientemente descrita de forma a ser replicada e se estava adequada ao alcance dos objetivos; se os resultados eram compatíveis com a metodologia utilizada, merecendo credibilidade e se a aplicabilidade dos resultados é possível na prática, sendo que os benefícios mostram-se superiores aos riscos potenciais e justificam os custos ${ }^{(19)}$.

O Teste de Relevância III ou Final foi realizado pela análise da íntegra dos artigos, utilizando o Cheklist for measuring study quality ${ }^{(15)} \mathrm{e}$ a escala de Jadad $^{(16)}$ para avaliação metodológica dos estudos, 
Figura 1 - Fluxograma das etapas do processo de seleção de estudos. Porto Alegre, RS, maio de 2009 a maio de 2011.

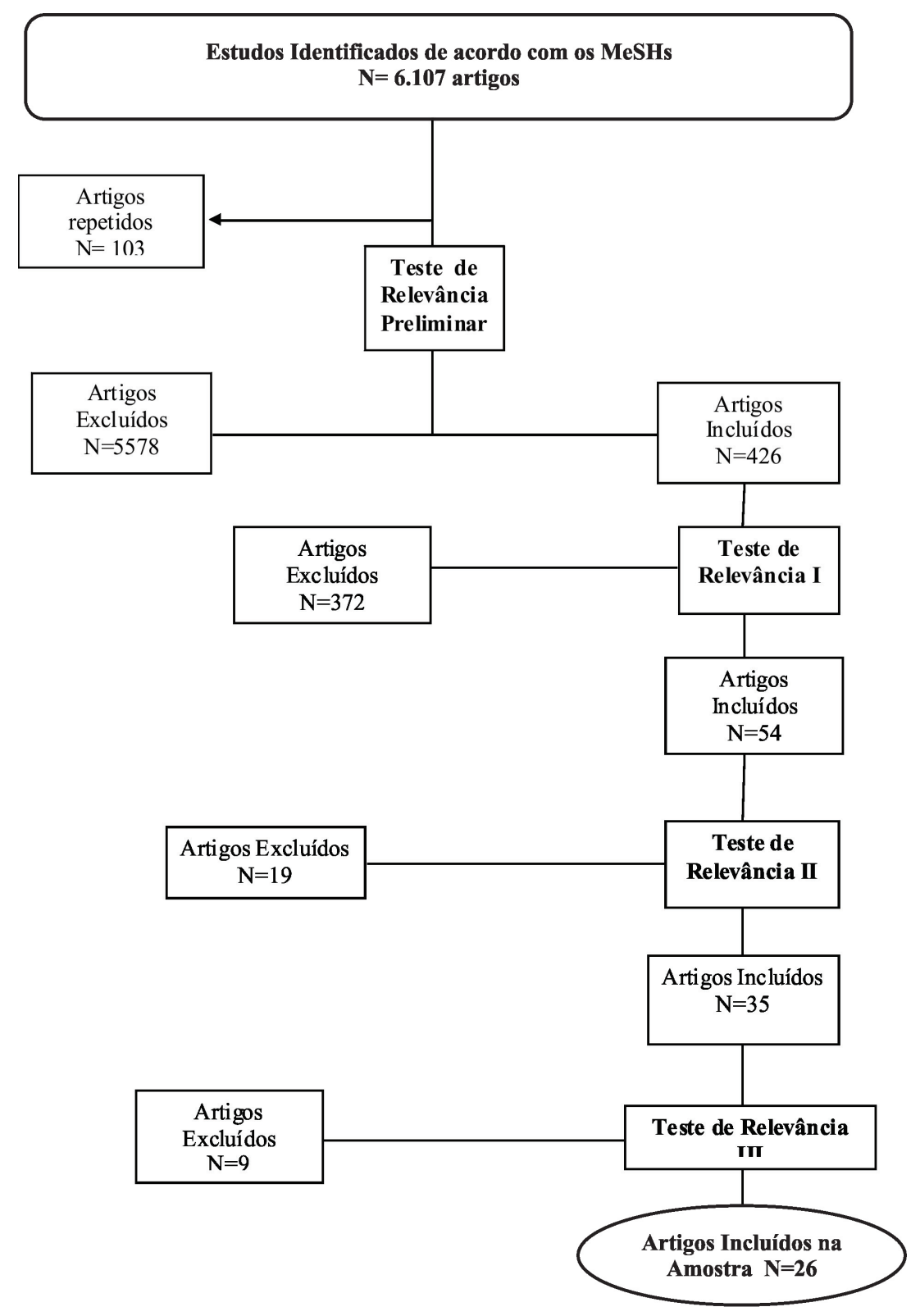

conferindo maior fidedignidade à qualidade dos mesmos. Ao final do Teste de Relevância III, decidiu-se excluir aqueles que tivessem nível de evidência maior que 4, score de qualidade pelo cheklist abaixo de 14 e escala de Jadad menor que 3. Assim, foram excluídos 9 artigos, compondo a amostra final 26 estudos.

A coleta de dados foi relacionada com a pergunta norteadora da revisão sistemática. Os resultados foram extraídos pela pesquisadora, sendo registrados em um quadro sinóptico, no qual foram incluídas informações detalhadas de cada artigo, tais como: autor, tipo de estudo, dados que caracterizam os sujeitos, metodologia e descrição do curativo, evidência dos resultados, aplicabilidade dos mesmos, vantagens e desvantagens.

A discussão dos resultados foi focada nas implicações das evidências apresentadas em relação ao curativo prevalente utilizado no tratamento de complicações de FOs abdominais. Mediante análise e conjunto dos estudos incluídos na revisão, foi atribuído ao tratamento para complicação de FO 
abdominal uma classificação quanto ao nível de evidência e ao grau de recomendação.

Nesta revisão sistemática, os níveis de evidências e grau de recomendação utilizados foram: Níveis de Evidências : 1. Revisão Sistemática; 2. Ensaio Clínico Randomizado; 3. Coorte; 4. Caso Controle. Graus de Recomendação: A) Resultado recomenda a intervenção - Evidência forte; B) Resultado não é conclusivo - não é suficiente para confirmar a hipótese - evidência Moderada; C) Resultado contraindica a intervenção - evidência fraca ${ }^{(17)}$.

O grau de recomendação da evidência foi hierarquizado, utilizando-se as letras do alfabeto e escritas maiúsculas junto com o nível de evidência ${ }^{(13)}$.

\section{RESULTADOS}

A amostra foi composta por 26 artigos, sendo 1 revisão sistemática (3,84\%), 7 ensaios randomizados (26,92\%), 16 coortes $(61,53 \%)$ e 2 caso-controle (7,69\%). Em relação ao país de origem, houve uma predominância de estudos realizados nos EUA, com 8 artigos $(30,76 \%)$.

A base de dados que prevaleceu na amostra foi a Scopus, com 8 artigos (30,76\%). Já a base de dados Cochrane não apresentou nenhum artigo que respondesse a questão norteadora. $\mathrm{O}$ tipo de cirurgia prevalente foi a Laparotomia, com 11 pesquisas $(42,30 \%)$ seguida de cirurgia de colonretal e retossigmoidectomias, com 5 (19,23\%). A cirurgia de correção de hérnia incisional foi a menos prevalente, com apenas 1 estudo (3,84\%).

Em relação aos curativos, foram encontrados 11 tipos diferentes. Destes, quatorze avaliaram o uso do curativo a vácuo com pressão negativa, mais conhecido como VAC ou TNP (terapia de pressão negativa), e dois analisaram o VAC associado a outro tipo de curativo.

Considerando os componentes para a formulação da questão de pesquisa $(\mathrm{P}=$ participante, $\mathrm{I}=$ intervenção e $\mathrm{O}=$ desfecho), entende-se ser importante explicitar de forma mais detalhada o curativo a VAC, bem como seu mecanismo de ação, indicação de uso, trocas e tempo de cicatrização da ferida.

\section{DISCUSSÃO}

O VAC ou TNP é um curativo que usa subpressão atmosférica, ou pressão negativa, o qual aumenta a perfusão dermal, estimula a formação de tecido de granulação, reduz edema e o fluido intersticial do tecido, estimula a formação de tecido reverso e reduz a colonização bacteriana. É indicado quando o fechamento fascial primário não é possível entre o sétimo e décimo dia, e corre-se o risco de vísceras aderirem na parede abdominal e a fáscia retrair. Tem habilidade de atuar como um efetivo enxerto de pele sobre superfícies irregulares, sendo indicado em abdômen aberto, ferida no esterno, defeitos de tecidos moles, trauma, fascite necrosante, fixação de enxerto de pele e queimaduras. É contraindicado em feridas agudas, na presença de tecido necrótico, infecção invasiva maciça, exposição cortical óssea, sangramento ativo de um sítio de ferida ${ }^{(18)}$.

O VAC é composto de espuma com um poro de $400-600 \mu \mathrm{m}$ ( V.A.C Pack curativo) de poliuretano preta reticulado ou álcool polivinil e uma pressão negativa contínua ou intermitente, portanto, especialmente adequado a feridas de exudato intenso ${ }^{(19)}$. A interação da espuma e tecido sob pressão negativa contínua resulta em macro e micro deformações, as quais estimulam a formação do tecido de granulação e neovascularização ${ }^{(20)}$.

A pressão negativa indicada variou bastante. Houve indicação de $-50 \mathrm{mmHg}{ }^{(21)},-75 \mathrm{mmHg}$ a $-125 \mathrm{mmHg}^{(22-26)},-80 \mathrm{mmHg}{ }^{(27)},-100$ a $-150 m m \mathrm{Hg}^{(28)}$, $-175 \mathrm{mmHg}^{(29)}$. Assim, percebe-se que a pressão negativa que prevaleceu foi $-75 \mathrm{mmHg}$ a $-125 \mathrm{mmHg}$.

A troca de curativos foi indicada a cada $48-72$ horas pelos estudos ${ }^{(18,17,23,24,25,27)}$. Outros dois artigos indicaram trocas a cada 72-120 horas ${ }^{(22,29)}$ e um indicou troca a cada 24-72 $\mathrm{h}^{(30)}$. Contudo, ressalta-se que estudos com nível de evidência e grau de recomendação $1 \mathrm{~A}^{(31)}$ e $2 \mathrm{~A}^{(32)}$ orientaram trocas de 48 a 72 horas.

O tempo de cicatrização, que foi o desfecho proposto nesta revisão, $\mathrm{O}=$ tempo de cicatrização, foi o dado que mais variou. Provavelmente, devido à heterogenidade dos tipos de feridas tratadas com o VAC em cada um dos estudos. Assim, destaca-se um deles ${ }^{(18)}$, com nível de evidência $1 \mathrm{~A}$, o qual apontou tempo médio de cicatrização entre 7 a 49 dias e outro $2 \mathrm{~A}^{(19)}$, o qual referiu 7 dias de cicatrização. Em outro artigo ${ }^{(20)}$, o tempo de cicatrização foi em média 29 dias, sendo 31 para deiscência de FO e 25 dias para FO abdominal. Já uma quarta pesquisa $^{(21)}$ apontou 44 dias para deiscência de FO e 40 dias para FO abdominal. A média de tempo apontada pelas demais produções variou de 4,4 $\operatorname{dias}^{(22)}$ a $21 \operatorname{dias}^{(29)}$, com média de $18 \operatorname{dias}^{(23)}, 15,4$ $\operatorname{dias}^{(26)}$ e $13,2 \operatorname{dias}^{(21)}$. 
Ressalta-se, que apenas dois estudos com nível de evidência elevado, do tipo 1 e 2 , avaliaram a eficiência do $\mathrm{VAC}^{(18,19)}$. Nos demais que realizaram esta avaliação, o nível de evidência é mais baixo (14 coorte e um caso controle). Todos os estudos encontraram resultados positivos ${ }^{(20-3)}$, entretanto destacaram a importância de se realizar pesquisas do tipo randomizado, testando a eficácia do VAC, com amostra maior de pacientes.

\section{CONCLUSÃO}

Esta revisão apresentou como prevalente a descrição de 16 estudos utilizando o curativo a vácuo ou terapia de pressão negativa como terapia única e que combinaram a terapia a VAC com outros métodos de cobertura, sendo, sem dúvida, indicativo de uma tecnologia aplicada para tratamento de diferentes feridas, inclusive de FOs abdominais. Porém, os mesmos salientaram a necessidade do desenvolvimento de novas pesquisas, com nível de evidência elevada, do tipo revisão sistemática e ensaios clínicos randomizados com a terapia de pressão negativa em populações diferentes.

A presente pesquisa traz contribuições importantes ao conhecimento na área de tratamento de feridas. É a primeira em âmbito mundial, com modelo de revisão sistemática, que disserta sobre o curativo ideal para tratamento de FOs abdominais com complicações. Pontuou-se, neste artigo, a existência de evidência forte para o curativo VAC.

Espera-se, com esta revisão, poder contribuir e subsidiar o desenvolvimento de um protocolo clínico voltado ao tratamento de FO abdominal, que, posteriormente, deverá ser validado.

Neste sentido, sugerem-se novas pesquisas, como ensaio clínico randomizado, ou estudo de caso controle, para que se possa avaliar a efetividade e a viabilidade da terapia VAC na nossa realidade.

\section{REFERÊNCIAS}

1 Dealey C. Cuidando de Feridas: um guia para enfermeiras. $2^{\text {a }}$ ed. São Paulo: Atheneu; 2001.

2 Hospital de Clínicas de Porto Alegre. Business Intelligence Gestão de Performance IBSC. Cubo produção assistencial. Porto Alegre: HCPA, [2007].
3 Carr KC. Developing an evidence-based practice protocol: implications for midwifery practice. J Midwifery Women's Health. 2000;45(6):544-51.

4 Barbosa H, Amâncio A.Controle clínico do paciente cirúrgico. 4ª Ed. Rio de Janeiro: Atheneu; 2009.

5 Odom-Forren J. Preventing surgical site infections. Nurs. 2006;36(6):58-63.

6 Jorge SA, Dantas SRPE. Abordagem multiprofissional do tratamento de feridas. São Paulo: Atheneu; 2005.

7 Haddad MCL, Bruschi LC, Martins EAP. Influência do açúcar no processo de cicatrização de incisões cirúrgicas infectadas. Rev Latino-Am Enferm. 2000;8(1):57-65.

8 Porchat CA, Santos EG, Neto GPB. Complicações pós-operatórias em pacientes submetidos à abdominoplastia isolada e combinada à outras cirurgias do abdome. Rev Col Bras Cir. 2004;31(6):368-372.

9 Mizell JS. Abdominal surgical incisions: prevention and treatment of complications [Internet]. Waltham: Uptodate; 2011 [cited 2011 jul 17] . Available from: http:// www. uptodate.com/contents/abdominal-surgical-incisions-prevention-and-treatment-of-complications

10 Fernandez OS, Díaz JM, González AC, Benavides RB, Castillo JZ. Factores de riesgo pra dehiscencia de herida quirúrgica. Cir Cir. 2000;68(5):198-203.

11 Ferreira AM, Andrade D. Sítio cirúrgico: avaliação e intervenção de enfermagem no pós-operatório. Arq Ci Saúde. 2006;13(1):27-33.

12 Silva CG. Curativos para tratamento de deiscência de feridas operatórias abdominais: uma revisão sistemática [dissertação]. Porto Alegre: Escola de Enfermagem da Universidade Federal do Rio Grande do Sul; 2011.

13 Pereira AL, Bachion MM. Atualidades em revisão sistemática de literatura, critérios de força e grau de recomendação de evidência. Rev Gaúcha Enferm. 2006;27(4):491-498,

14 Richardson WS, Wilson MC, Nishikawa J, Hayward RSA. The well-built clinical question: a key to evidence-based decisions. ACP J Club. 1995;123(3):A 12-3

15 Downs SH, Black N. The feasibility of creating a checklist for the assessment of the methodological 
quality both of randomized and non-randomised studies of health care interventions. J Epidemiol Community Health. 1998;52(6):377-384.

16 Clark HD, Wells GA, Huet C, McAlister FA, Salmi LR, Fuguston D, et al. Assessing the quality of randomized trials: reability of the Jadad scale. Control Clin Trials. 1999;20(5):448-52.

17 Sampaio RF, Mancini MC. Estudos de revisão sistemática: um guia para síntese criteriosa da evidência científica. Rev Bras de Fisioterapia. 2007;11(1):83-89.

18 Bovill E, Banwell PE, Teot L, Eriksson E, Song C, Mahoney J, et al. Topical negative pressure wound therapy: a review of its role and guidelines for its use in the management of acute wounds. Int Wound J. 2008;5(4):511-528.

19 Mouës CM, Van den Bemd GJCM, Heule F, Hovius SER. Comparing conventional gauze therapy to vacuum-assisted closure wound therapy: a prospective randomized trial. J Plast Reconstr Aesthet Surg. 2007; 60(6):672-681.

20 Trueman P, Flack S, Loonstra A, Hauser T. The feasibility of using V.A.C. Therapy in home care patients with surgical and traumatic wounds in the Netherlands. Int Wound J. 2008;5(2):225-231.

21 Gabriel A, Shores J, Heinrich C, Baqai W, Kalina S, Sogioka N, Gupta S. Negative pressure wound therapy with instillation: a pilot study describing a new method for treating infected wounds. Int Wound J. 2008;5(3):399-413.

22 Mayer D, Rancic Z, Meier C, Pfammatter T, Veith FJ, Lachat M. Open abdomen treatment following endovascular repair of rupture abdominal aortic aneurysms. J Vasc Surg. 2009;50(1):1-7.

23 Brox-Jiménez A, Díaz-Gómez D, Parra-Membrives P, Martínez-Baena D, Márquez-Muñoz M, Lorente-Herce J, et al. Sistema de cierre asistido por vacío em heridas complejas. Estudio retrospectivo. Cir Esp. 2010;87(5):312-317.

\section{Endereço da autora / Dirección del autor / Author's address}

Carolina Giordani Silva

Av. Bento Gonçalves, $n^{\circ} 1515$, ap. 607B, Bairro Partenon

90650-002, Porto Alegre, RS.

E-mail: carol.giordani@gmail.com ou cgsilva _0701@hotmail.com
24 Amin AI, Shaikh IA. Topical negative pressure in managing severe peritonitis: A positive contribution? World J Gastroenterol. 2009;15(27):3394-3397.

25 Heller L, Levin SL, Butler CE. Management of abdominal wound dehiscence using vacuum assisted closure in patients with compromised healing. Am J Surg. 2006;191(2):165-172.

26 Labler L, Trentz O. The use of vacuum assisted closure (VACTM) in soft tissue injuries after high energy pelvic trauma. Langenbecks Arch Surg. 2007;392(5):601-609.

27 Campbell PE, Smith GS, Smith JM. Retrospective clinical evaluation of gauze-based negative pressure wound therapy. Int Wound J. 2008;5(2):280-286.

28 Barker DE, Green JM, Maxwell RA, Smith PW, Mejia VA, Dart BW et al. Experience with vacuum-pack temporary abdominal wound closure in 258 Trauma and general and vascular surgical patients. J Am Coll Surg. 2007;204(5):784-791.

29 Miller PR, Meredith JW, Johnson JC, Chang MC Prospective evaluation of vacuum-assisted fascial closure after open abdomen. Annals of Surg. 2004;239(5): 608-616.

30 Batacchi S, Natano S, Nella A, Zagli G, Bonizzoli M, Pasquini A et al. Vacuum-assisted closure device enhances recovery of critically ill patients following emergency surgical procedures. Crit Care. 2009;13(6):1-8.

31 Gäddnäs F, Saarnio J, Ala-Kokko T, Laurila J, Koivukangas V. Continuous retention suture for the management of open abdomen: a high rate of delayed fascial closure. Scandinavian J Surg. 2007;96(4):301-307.

32 Gracias VH, Braslow B, Johnson J, Pryor J, Gupta R, Reilly P, et al. Abdominal Compartment Syndrome in the Open Abdomen. Arch Surg. 2002;137(11):1298-1300.

33 Jamshidi R, Schecter WP. Biological dressings for the management of enteric fistulas in the open abdomen. Arch Surg. 2007;142(8):793-796

Recebido em: 09.12.2011

Aprovado em: 12.07.2012 